\title{
Step-Lattice-Induced Band-Gap Opening at the Fermi Level
}

\author{
F. Baumberger, ${ }^{1, *}$ M. Hengsberger, ${ }^{1}$ M. Muntwiler, ${ }^{1}$ M. Shi, ${ }^{2}$ J. Krempasky, ${ }^{2}$ L. Patthey, ${ }^{2}$ J. Osterwalder,,${ }^{1}$ and T. Greber ${ }^{1}$ \\ ${ }^{1}$ Physikinstitut der Universität Zürich, Winterthurerstrasse 190, CH-8057 Zürich, Switzerland \\ ${ }^{2}$ Swiss Light Source, Paul Scherrer Institut, CH-5232 Villigen, Switzerland
}

(Received 8 September 2003; published 9 January 2004)

\begin{abstract}
The interaction of the Shockley surface state with the step lattice of vicinal $\mathrm{Cu}(111)$ leads to the formation of an electronic superlattice state. $\mathrm{On} \mathrm{Cu}(443)$, where the average terrace length forms a "shape resonance" with the Fermi wavelength, we find a step-lattice-induced band-gap opening at the Fermi level. A gap magnitude $>200 \mathrm{meV}$ is inferred from high resolution photoemission experiments and line shape analysis. The corresponding energy gain with respect to a gapless case is $\approx 11 \mathrm{meV} /$ unit cell, and is a substantial contribution to the stabilization of the step lattice.
\end{abstract}

DOI: 10.1103/PhysRevLett.92.016803

The stability of vicinal surfaces is governed by the behavior of the temperature dependent anisotropic surface tension $\gamma$, which in turn depends on step and step interaction energies $[1,2]$. Both contributions to $\gamma$ have non-negligible electronic components, which are generally poorly understood. The investigation of vicinal surfaces, where steps form a dense one-dimensional (1D) lattice, may thus contribute to understanding a number of important material properties which are controlled by $\gamma$, such as crystal growth, surface roughening, or the equilibrium shape of small crystallites $[3,4]$. The dominant contribution to $\gamma$ is the formation energy of single isolated steps. Interactions between steps are typically 2 orders of magnitude smaller [3]. Nonetheless, they can crucially influence the behavior of surfaces, for example, by rendering particular surface orientations unstable, with respect to faceting in different vicinal faces [5]. Furthermore, they determine the degree of order on a vicinal surface. Vice versa, they can be obtained from the width and shape of the terrace width distribution [6].

Step-step interactions have typically been attributed to elastic interactions due to the lattice relaxation around step sites, and to dipole-dipole forces caused by the charge rearrangement at step sites [4]. Both scale with the inverse square of the terrace length $L$, thus leading to monotonically increasing disorder in the step lattice as the terrace length increases. More diverse behaviors, such as extrema in the roughening temperature for "magic" terrace lengths, may be expected in the presence of electronic step-step interactions. Such interactions are mediated by the conduction electrons, reacting to the structural changes on the surface [7,8]. The primary candidates for long ranged electronic interactions are metallic surface states, since the decay of Friedel oscillations in the charge density with distance from the scatterer diminishes from $r^{-5}$ for 3D states to $r^{-2}$ for the intrinsically 2D surface states [8].

On $\mathrm{Cu}(111)$ the Shockley surface state is metallic, though it has only an occupancy of about $1 / 25$ of an electron per surface unit cell and is thus not expected to
PACS numbers: 73.20.At, 68.35.Md, 73.21.Cd, 79.60.-i

play a dominant role in the energetics of the system [9]. However, it was shown for the homoepitaxial system of $\mathrm{Cu}$ on $\mathrm{Cu}(111)$ that adatoms at low temperatures preferentially arrange with interatomic distances of $n \lambda_{F} / 2$, where $\lambda_{F} \approx 2.9 \mathrm{~nm}$ is the Fermi wavelength of the surface state $[10,11]$. In a one-dimensional, periodic system with a large unit cell, surface state mediated adsorbate interactions were inferred even at room temperature [12]. On vicinal surfaces, the unit cells are intrinsically large, and the scatterers one dimensional. We thus expect surface states to influence energy balance and surface structure. Electronic step-step interactions on vicinal surfaces have been predicted for several cases based on model potential calculations $[13,14]$. However, only indirect experimental evidence for their presence has been reported $[15,16]$. In particular, a direct measurement of the superlattice related modification in the electronic band dispersion around the Fermi level has been lacking so far. In this Letter, we report the observation of a step-lattice-induced band gap, opening at the Fermi level in the surface state dispersion of $\mathrm{Cu}(443)$. This lowers the energy of the electronic system, resulting in a step-lattice stabilization for $L \approx \lambda_{F} / 2$.

Surface states on metal surfaces are widely investigated as model systems for the behavior of electrons in nanostructures [17,18]. In order to understand surface state mediated interactions on vicinal surfaces, it is essential to determine the scattering properties of steps. However, controversial results have been reported. Scanning tunneling microscopy (STM) and photoemission studies of similar surface states on large $\mathrm{Ag}(111)$ and $\mathrm{Au}(111)$ terraces indicated zero transmission across step edges, resulting in total confinement on single terraces and an energy shift of the lowest lying state $\propto L^{-2}$, consistent with a particle in a box picture [18-20]. Photoemission experiments on vicinal $\mathrm{Cu}$ with terrace lengths $L \lesssim 15 \AA$, on the other hand, found propagating free-electron-like states as inferred from the roughly parabolic band dispersion and from the shift in binding energy $\propto L^{-1}[21-25]$. 


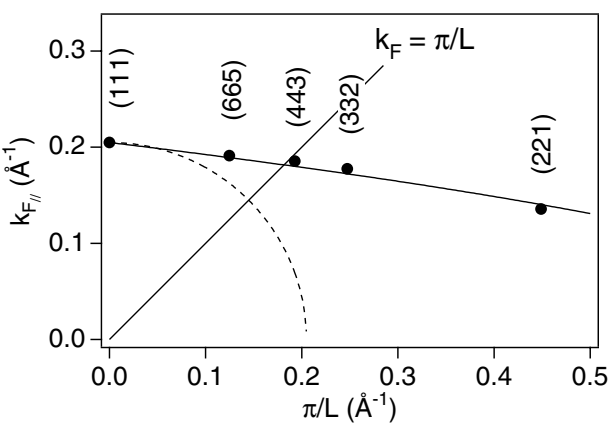

FIG. 1. Measured surface state Fermi wave vectors $k_{F_{\|}}$parallel to the step direction for several vicinal $\mathrm{Cu}(111)$ surfaces. The solid line is a fit to a Kronig-Penney model. The dashed line shows $k_{F_{\|}}$for electrons confined in a lateral quantum well of width $L$.

Figure 1 shows surface state Fermi wave vectors measured parallel to the steps on several vicinal $\mathrm{Cu}(111)$ surfaces, investigated for this work. The data follow the behavior expected for quasifree electrons, propagating in a weak 1D periodic potential (solid line). The deviation from a particle in a box picture (dashed line), where the surface state depopulates at $\lambda_{F}^{(111)} / 2$, is significant. If the above band structure picture describes the dispersion, we expect the opening of a gap at the Fermi level if the Fermi surfaces of subsequent surface Brillouin zones touch. From Fig. 1 it is seen that this shape resonance condition $\left(k_{F}=\pi / L\right)$ is best met for $\mathrm{Cu}(443)$. However, several earlier investigations on vicinal $\mathrm{Cu}(111)$ surfaces gave no indications of a step-lattice-induced band gap $[22,23]$. Our systematic photon-energy dependent studies resolve this issue. The band gap is hidden for most measurement conditions by the large inhomogeneous broadening of the photoemission spectra and reveals itself only for photon energies close to the cross section minimum around $50 \mathrm{eV}$, where states from consecutive Brillouin zones have similar weights [26].

$\mathrm{Cu}(443)$ is a $B$-type vicinal $\mathrm{Cu}(111)$. Its nominal surface structure consists of monatomic steps, with (111) microfacets, separated by $7 \frac{1}{3}$ atomic rows wide fcc(111) terraces. A well ordered surface was prepared with standard procedures and oriented in situ with low energy electron diffraction (LEED) [24]. The LEED pattern showed a clear spot splitting, consistent with the nominal step superlattice vector $Q=2 \pi / L^{(443)}$, where $L^{(443)}=16.3 \AA$. The terrace width distribution was estimated from the spot profile to have a standard deviation $\leq 0.36 \mathrm{~L}$. Photoemission data have been taken at room temperature with a Scienta 2002 analyzer at the SIS beam line of the Swiss Light Source (SLS). The resolution was set to $\approx 25 \mathrm{meV} / 0.3^{\circ}$ (FWHM) in energy/angle.

Figure 2(a) shows a Fermi surface map from $\mathrm{Cu}(443)$, recorded with $h \nu=53 \mathrm{eV}$. The wave vector component $k_{x}$ points in the direction perpendicular to the steps. The Fermi surface resembles two free-electron-like,
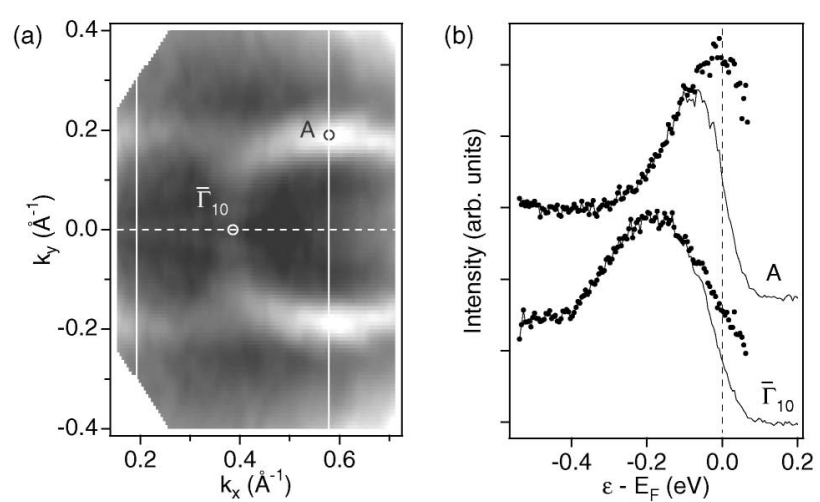

FIG. 2. (a) Fermi surface map from $\mathrm{Cu}(443)$, measured with $h \nu=53 \mathrm{eV}$. The solid white lines mark the zone boundaries of the step lattice. (b) Spectra at two different points in the Brillouin zone, as labeled in (a). Raw data are shown as thin lines. The dots result after normalization with a Fermi-Dirac function.

quasicircular contours, offset by a reciprocal lattice vector of the step lattice. This is direct evidence for the formation of a superlattice state. The Fermi surface cannot be explained by final state umklapp processes, since no similar effects are observed for the equally intense bulk $s p$ bands (not shown). Hence, the energy density in the surface state must depend on the terrace length, and thus gives rise to an electronic step-step interaction. The coincidence of the contour centers with the zone boundaries indicates propagation of the surface state along the macroscopic surface plane, rather than the individual terraces $[21,23]$. At the zone center $\left(\bar{\Gamma}_{10}\right)$ the two contours merge by opening a neck along the symmetry line. The depletion of spectral weight at the merging point indicates the opening of a band gap. Its location at the $\bar{\Gamma}$ points reflects the significant $p_{x}$ symmetry of the surface state in a step lattice. The observation of a band gap is confirmed by the energy distribution curves (EDC's) shown in Fig. 2(b). The EDC taken at $\bar{\Gamma}_{10}$ shows a peak significantly below the Fermi level, clearly distinct from the spectrum taken at point A, where a Fermi level crossing is evident after normalization of the spectra with the Fermi-Dirac distribution.

In order to be more quantitative, we have measured the dispersion of the surface state perpendicular to the steps for several photon energies. Data taken at $h \nu=46 \mathrm{eV}$ are shown in Fig. 3. Consistent with the data in Fig. 2, we observe a band in three Brillouin zones. Its dispersion follows the periodicity of the step lattice by being bent back at $\bar{\Gamma}_{10}$. The detailed behavior of the line shapes is better seen in the EDC's shown in Fig. 3(b). Clearly, peak widths and shapes strongly vary with momentum, an effect completely unexpected for a weakly interacting electronic system. Furthermore, the rather broad linewidth around $\bar{\Gamma}_{10}$ complicates a direct quantification of the gap magnitude. To clarify these issues we performed a 

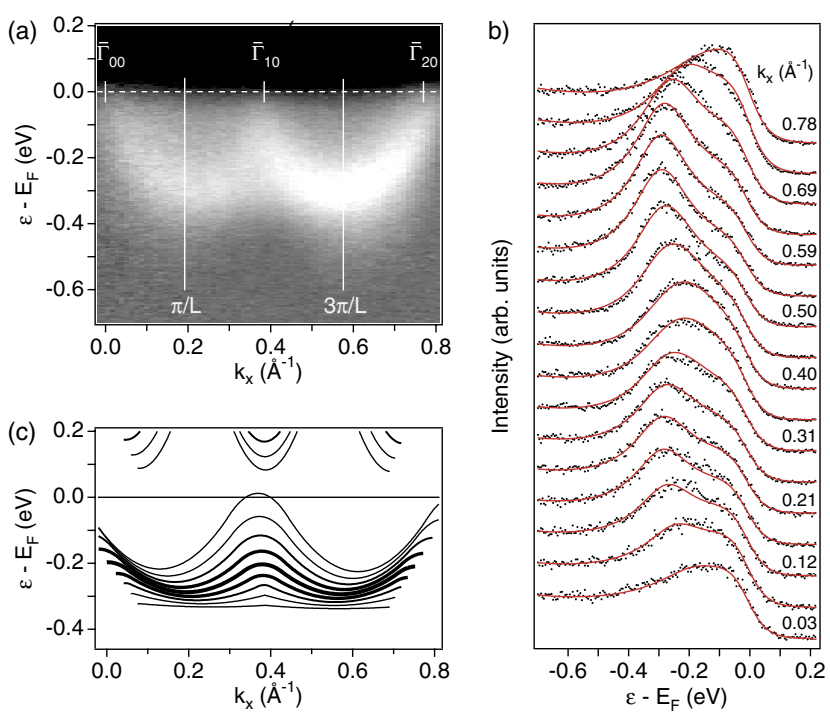

FIG. 3 (color online). Surface state dispersion perpendicular to the steps. (a) Gray scale plot of raw data with zone boundaries and symmetry points overlaid. (b) Subset of EDC's from the same data, together with the corresponding profiles from the 2D fit (for details see text). (c) Kronig-Penney band structures, weighted with their importance for $\mathrm{Cu}(443)$.

line shape analysis of the photoemission data, aiming as well to justify the assignment of the peak to a single band. To do so, we used a model similar to the one published in Ref. [24]. The physical idea behind it is as follows. The surface state has a rather short coherence length $\lambda_{c}$, presumably in the range of only a few terrace widths. Thus the photoemission intensity has to be described as the incoherent sum over emission from areas limited in linear dimension by $\lambda_{c}$. These areas, in general, have different local structures, since step positions fluctuate around the nominal lattice positions. For simplicity we assign to each of these areas a Kronig-Penneytype dispersion which depends only on the average terrace length $\bar{L}$ in a given region. The dispersions are weighted with a probability distribution $P(\bar{L})$ which we assume to be Gaussian. The photoemission intensity is then calculated by numerically integrating the $P$-weighted spectral functions for all contributing $\bar{L}$.

The effects of this broadening are illustrated in Fig. 3(c), where we show a set of dispersion relations, weighted with their importance $P(\bar{L})$ for $\mathrm{Cu}(443)$. The largest energy spread of the dispersion curves coincides with the significant peak broadening around $\bar{\Gamma}_{10}$. Furthermore, it can be seen that the gap at $\bar{\Gamma}_{00}$ and $\bar{\Gamma}_{20}$ almost vanishes in the integrated intensity. For the same reason the band gap cannot be clearly resolved at low photon energies, where only the state centered at the first zone boundary $(\pi / L)$ is observed [26].

Two-dimensional fits to the data with the above described model show an almost quantitative agreement of fit function with the experimental line shapes, which we take as strong evidence for the assignment of the peak at
$\bar{\Gamma}_{10}$ to a single band bending back at the zone centers significantly below the Fermi level. Using the procedure described above, the analysis of data taken at various photon energies consistently yields the following results: the band bottom is located at $320 \pm 15 \mathrm{meV}$ below the Fermi level, and the bandwidth is found to be in the range of 90-140 meV. This leaves a gap of about $200 \mathrm{meV}$ below $E_{F}$, and the full gap size is estimated to be as large as $450 \mathrm{meV}$.

In the following we discuss the surface state contribution to the stability and order of the surface. First, we note that the energy balance of vicinal metal surfaces is extremely delicate. The most recent calculations of Raouafi et al. give an energy difference between $B$-type vicinal $\mathrm{Cu}(111)$ and a surface faceted in (111) and (110) faces of only $\approx 45 \mathrm{meV} /$ unit cell of the vicinal surface [ $\approx 1.2 \mathrm{meV} / \AA^{2}$ for $\mathrm{Cu}(443)$ ] [5]. Earlier investigations even reported the opposite sign; i.e., they claimed the instability of most vicinal surfaces at low temperatures [1]. These values are comparable with the energy density $u$ of the surface state, given by $u^{(111)}=\int_{-\infty}^{\epsilon_{F}}(\epsilon-$ $\left.\epsilon_{B}\right) g_{f}(\epsilon) d \epsilon \approx 7.3 \mathrm{meV} /$ unit cell $\left(\approx 1.3 \mathrm{meV} / \AA^{2}\right)$, where $g_{f}$ is the constant DOS of the 2D free electron gas on $\mathrm{Cu}(111)$ with binding energy $\epsilon_{B}$.

The opening of a band gap lowers the energy in the surface state. The corresponding change in surface tension $\Delta \gamma$ can be estimated by comparing the energy density of the superlattice state with a nongapped state with the same occupation [9]:

$$
\Delta \gamma(L)=\int_{-\infty}^{\epsilon_{F}}\left[\left(\epsilon-\epsilon_{B}^{s l}\right) g_{s l}(\epsilon, L)-\left(\epsilon-\epsilon_{B}^{f}\right) g_{f}(\epsilon)\right] d \epsilon,
$$

where $g_{s l}$ is the DOS for the superlattice band. In order to conserve the total charge, we allow for slightly different binding energies $\epsilon_{B}^{s l}$ and $\epsilon_{B}^{f}$ of the superlattice and the free-electron-like states.

Figure 4(a) shows the numerical evaluation of $g_{s l}$, for a Kronig-Penney-type band with a confining step potential barrier of $7 \mathrm{eV} \AA$ that was extracted from the experimental data in Fig. 3. It exhibits the characteristic van Hove singularity, caused by the saddle points of the dispersion at the zone boundaries [see inset of Fig. 4(a)]. The pileup of density of states at energies below the Fermi level reduces the energy, and thus contributes to the stability of the surface. The terrace length dependence of $\Delta \gamma$, calculated with the above potential barrier common to all surfaces, and a band onset given by $\epsilon_{B}^{s l}=\epsilon_{B}^{(111)}+$ $1.4 \mathrm{eV} \AA / L$ is shown in Fig. 4(b). These parameters give accurate descriptions of the band dispersions for all investigated surfaces. A pronounced minimum in $\Delta \gamma$ caused by the opening of a band gap is evident. It is slightly shifted from $L=\lambda_{F} / 2$, because the number of gapped states still increases beyond $\lambda_{F} / 2$ with increasing overlap of the free electron Fermi surfaces from consecutive Brillouin zones. The open symbols in 
(a)

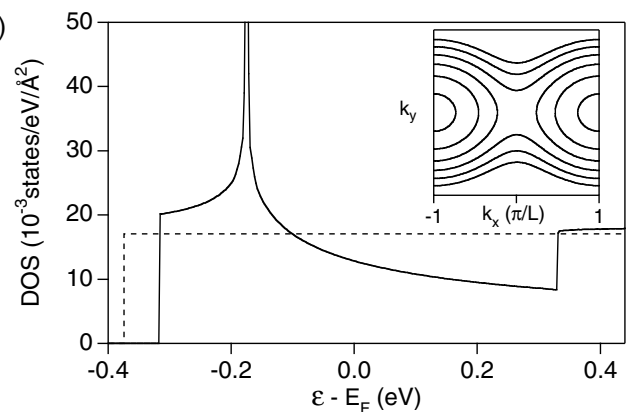

(b)

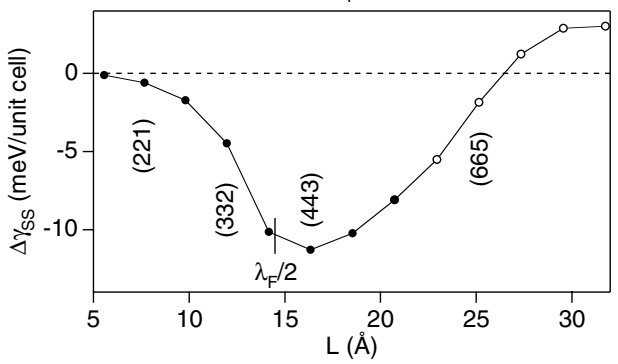

FIG. 4. (a) Density of states $g_{s l}(\epsilon)$ for a $2 \mathrm{D}$ electron gas that is confined in the $x$ direction by a potential barrier of $7 \mathrm{eVA}$. The constant DOS of a 2D free electron with the same effective mass and occupation is shown for comparison (dashed line). The inset shows a contour plot of isoenergy lines up to the Fermi energy. (b) Terrace-length dependent change of the surface tension due to the formation of a superlattice state.

Fig. 4(b) reflect the ambiguity in the character of the surface state wave function on longer terrace lengths $[23,26]$.

The maximal reduction of $\gamma$ is $\approx 11 \mathrm{meV} /$ unit cell which is substantial and can be expected to influence, e.g., the roughening temperature. The enhanced stability of intermediate terrace lengths might also cause signatures in the terrace width distribution, which can be measured by various techniques. To our knowledge, there are no systematic experimental studies of roughening temperature or terrace width distributions on $B$-type vicinal $\mathrm{Cu}(111)$ available. A recent STM study reporting the structure of the related $A$-type [(100) step facets] surfaces concentrated on a shorter $(10.2 \AA)$ and much longer $(32.3 \AA, 47.8 \AA)$ terrace lengths than studied here, and no deviations from the $1 / L^{2}$ scaling of the terrace width distribution were observed [27]. Since these average terrace lengths do not meet the condition $k_{F} \approx$ $\pi / L$, the results obtained in Ref. [27] are not in contradiction to ours. Moreover, the non-negligible surface state contribution demonstrates that only the most accurate surface electronic structure calculations are suitable to discuss the stability of vicinal surfaces.

In conclusion, we have reported the first observation of a step-lattice-induced band gap in a metallic surface state on a vicinal surface. The band-gap-induced reduction in the surface free energy is estimated with a simple model to be of the order of $10 \mathrm{meV} /$ unit cell. We expect that the minimum in $\gamma$ around terrace lengths of half of the surface state Fermi wavelength leads to an enhanced roughening temperature. It might also be observed in the terrace width distribution which can be measured by STM.

We thank L. Barbier, M. Giessen, N. J.C. Ingle, A. Kara, R. Monnier, and K. M. Shen for fruitful discussions. This work was performed at the Swiss Light Source, Paul Scherrer Institute, Villigen, Switzerland. Financial support by the Swiss National Science Foundation is gratefully acknowledged.

*Electronic address: baumberger@stanford.edu Present address: Department of Applied Physics, Stanford University, Stanford, CA 94305, USA.

[1] J.W. M. Frenken and P. Stoltze, Phys. Rev. Lett. 82, 3500 (1999).

[2] M. C. Desjonquères, D. Spanjaard, C. Barreteau, and F. Raouafi, Phys. Rev. Lett. 88, 056104 (2002).

[3] Z.-J. Tian and T. S. Rahman, Phys. Rev. B 47, 9751 (1993).

[4] M. Giesen, Prog. Surf. Sci. 68, 1 (2001).

[5] F. Raouafi, C. Barreteau, D. Spanjaard, and M.C. Desjonquéres, Phys. Rev. B 66, 045410 (2002).

[6] X.-S. Wang et al., Phys. Rev. Lett. 65, 2430 (1990).

[7] K. H. Lau and W. Kohn, Surf. Sci. 75, 69 (1978).

[8] A. Bogicevic et al., Phys. Rev. Lett. 85, 1910 (2000).

[9] N. Memmel, Surf. Sci. Rep. 32, 91 (1998).

[10] J. Repp et al., Phys. Rev. Lett. 85, 2981 (2000).

[11] N. Knorr et al., Phys. Rev. B 65, 115420 (2002).

[12] S. Lukas, G. Witte, and Ch. Wöll, Phys. Rev. Lett. 88, 028301 (2002).

[13] A. C. Redfield and A. Zangwill, Phys. Rev. B 46, 4289 (1992).

[14] Wei Xu, James B. Adams, and T. L. Einstein, Phys. Rev. B 54, 2910 (1996).

[15] J. Frohn et al., Phys. Rev. Lett. 67, 3543 (1991).

[16] W.W. Pai, J.S. Ozcomert, N.C. Bartelt, and T. L. Einstein, Surf. Sci. 307-309, 747 (1994).

[17] M. F. Crommie, C. P. Lutz, and D. M. Eigler, Nature (London) 363, 524 (1993).

[18] A. Mugarza et al., Phys. Rev. Lett. 87, 107601 (2001).

[19] L. Bürgi et al., Phys. Rev. Lett. 81, 5370 (1998).

[20] K. Morgenstern, K. F. Braun, and K. H. Rieder, Phys. Rev. Lett. 89, 226801 (2002).

[21] A. P. Shapiro, T. Miller, and T.-C. Chiang, Phys. Rev. B 38, 1779 (1988).

[22] O. Sánchez et al., Phys. Rev. B 52, 7894 (1995).

[23] J. E. Ortega et al., Phys. Rev. Lett. 84, 6110 (2000).

[24] F. Baumberger, T. Greber, and J. Osterwalder, Phys. Rev. B 64, 195411 (2001).

[25] F. Baumberger, T. Greber, B. Delley, and J. Osterwalder, Phys. Rev. Lett. 88, 237601 (2002).

[26] F. Baumberger et al. (to be published).

[27] M. Giesen and G. Schulze Icking-Konert, Surf. Rev. Lett. 6, 27 (1999). 